Pacific Journal of Mathematic 


\title{
POLYNOMIALS IN DENUMERABLE INDETERMINATES
}

\author{
RICHARD M. GRASSL
}

\begin{abstract}
D. Knuth used the Robinson-Schensted "insertion into a tableau" algorithm to give a direct 1-to-1 correspondence between "generalized permutations" and ordered pairs of generalized Young tableaux having the same shape. Since a generalized permutation characterizes a power product of differential indeterminates, the work of $D$. Mead on the principal differential ideal generated by a Wronskian provided an independent proof of the existence of the Knuth bijection. This work led Mead to suggest that other interesting combinatorial results may be found by equating the cardinalities of different vector space bases for the same finite-dimensional subspace of a differential ring. In a previous paper the author showed how such combinatorial identities follow from the study of "strong bases" for certain ideals in a ring of polynomials in a denumerable set of indeterminates. The present paper completes that work by presenting an infinite number of such strong bases and thus greatly expands the ring theory and differential algebra having applications in the enumeration of tableaux.
\end{abstract}

1. The ideals I. Let $R=F\left[y_{i j}\right]$ denote the polynomial ring in the algebraically independent indeterminates $y_{i j}(i=1,2, \cdots, n$; $j=0,1,2, \cdots)$ over a field $F$. In applications to differential algebra, one lets $y_{1}, y_{2}, \cdots, y_{n}$ be $n$ independent indeterminates and $y_{i j}$ be the $j$ th derivative of $y_{i}$. Then a principal differential ideal $[x]$ is the ideal $\left(x_{0}, x_{1}, x_{2}, \cdots\right)$ in which $x_{j}$ is the $j$ th derivative of $x$.

D. Mead's study in [9] of $\left[W_{n}\right]$, where $W_{n}$ is the Wronskian of $y_{1}, y_{2}, \cdots, y_{n}$, gives a vector space basis for $R$ consisting of determinantal products having a natural 1-to-1 correspondence with ordered pairs of Young tableaux of the same shape, having $n$ or fewer rows. Let $x_{n q}$ be the $q$ th derivative $\left(y_{1} y_{2} \cdots y_{n}\right)^{(q)}$; it is shown below that ideals $\left(x_{q}, x_{q+1}, \cdots\right)$, related to $\left[x_{n q}\right]$, share combinatorial properties with $\left[W_{n}\right.$ ] when $q=n(n-1) / 2$.

A combinatorial method for proving the existence of syzygies (i.e., the nonexistence of a strong basis) is also described. The structure of $\left(x_{0}, x_{1}, \cdots\right)$ is studied in a manner that gives the structures of all ideals generated by subsets of the $x_{j}$.

Let $\left\{x_{j}\right\}=x_{0}, x_{1}, \cdots$ be a finite or denumerable sequence in $R, I$ be the ideal $\left(x_{0}, x_{1}, \cdots\right)$, and $X$ be the set of all power products

$$
\xi=\left(x_{0}\right)^{a_{0}}\left(x_{1}\right)^{a_{1}} \cdots\left(x_{h}\right)^{a_{h}} ; \quad h, a_{i} \in\{0,1, \cdots\} .
$$


Let $A$ be a linearly independent (over $F$ ) subset of $R$ such that $L=\{\alpha \xi \mid \alpha \in A, \xi \in X\}$ generates the vector space $R$ over $F$; then it is easily seen that the subspace $I$ is generated by the subset $C$ of all $\alpha \xi$ in $L$ with $\xi \neq 1$. The set $A$ is called an $\alpha$-set for $\left\{x_{j}\right\}$ if $L$ is a basis for the vector space $R$; if $A$ is an $\alpha$-set, $C$ is a basis for $I$. If $L$ is not a basis for $R$, the linear dependence relations of the elements of $L$ are called syzygies. If $\left\{x_{j}\right\}$ has an $\alpha$-set, the sequence is said to be strong. Below we describe a family of sequences $\left\{x_{j}\right\}$, develop a number of $\alpha$-sets for each sequence, and for each $\alpha$-set give an algorithm for determining membership in the ideal $\left(x_{0}, x_{1}, \cdots\right)$.

A power product $(p p) \pi$ in the $y_{i j}$ of degree $d=\operatorname{deg} \pi$ and weight $w=$ wgt $\pi$ is a product of $d$ factors, each of which is one of the $y_{i j}$, with $w$ the sum of the second subscripts $j$ of these $d$ factors. Below, $Q=\left(q_{1}, q_{2}, \cdots, q_{n}, q_{n+1}\right)$ is an ordered $(n+1)$-tuple of fixed nonnegative integers, $q=q_{1}+q_{2}+\cdots+q_{n+1}, T=\left\{t_{1}, t_{2}, \cdots\right\}$ is a subset (not necessarily proper) of $\{q, q+1, \cdots\}$, and $\mu$ is a nonnegative real number. Whenever $\pi$ is written as $\pi=\rho \eta, \rho$ is the product of all the factors $y_{i j}$ of $\pi$ with $j<q_{i}$ and $\eta$ is the product of the factors $y_{i j}$ of $\pi$ with $j \geqq q_{i}$.

The set of all $p p$ in the $y_{i j}$ is designated as $P$. The word space is used to denote a vector space over $F$; thus $P$ is a space basis for the ring $R$.

For all $t$ in $T$, let $v_{t}$ be a linear combination with coefficients in $F$ of the $p p \pi=\rho \eta$ with

$$
\operatorname{deg} \pi+\operatorname{wgt} \pi \leqq n+t \text { and } \operatorname{deg} \eta+\mu \operatorname{wgt} \eta<n+\mu t
$$

and let $x_{t}$ be the sum of $v_{t}$ and a linear combination with nonzero coefficients in $F$ of all the products

$$
y_{1 j_{1}} y_{2 j_{2}} \cdots y_{n j_{n}} \text { with } j_{i} \geqq q_{i} \text { for } 1 \leqq i \leqq n \text { and } j_{1}+\cdots+j_{n}=t .
$$

Let $I=\left(x_{t_{1}}, x_{t_{2}}, \cdots\right)$ be the ideal in $R$ generated by the $x_{t}$ with $t$ in $T$.

2. Ordering of power products. Associated with the $\eta$ of a fixed $p p \pi=\rho \eta$ is a function $j(i, k)$ such that $\eta=\eta_{1} \eta_{2} \cdots \eta_{n}$ with either $\eta_{i}=1$ or

$$
\begin{aligned}
& \eta_{i}=y_{i j(i, 1)} y_{i j(i, 2)} \cdots y_{i j\left(i, d_{i}\right)} \\
& q_{i} \leqq j(i, k) \leqq j(i, k+1) \text { for } 1 \leqq k<d_{i}=\operatorname{deg} \eta_{i} .
\end{aligned}
$$

(If $\eta_{h}=1, j(i, k)$ is not defined for $i=h$.) This is next used to define nonnegative integers $g_{i}$, a function $M[i, k]$, and a sequence $\sigma(\pi)=s_{0}, s_{1}, \cdots$. Then $\sigma(\pi)$ will be used in a partial ordering of 
the $p p$ which is the key tool for the study of the structure of the ideal $I$.

Let $g_{1}=d_{1}$ and $M[1, k]=n(k-1)+1$ for $1 \leqq k \leqq g_{1}$. Now assume that $i>1$, that $g_{i-1}$ is defined, and that $M[i-1, k]$ is defined for $1 \leqq k \leqq g_{i-1}$. Let $g_{i}$ be the largest positive integer $m$ with $m+j(i, m)-q_{i} \leqq g_{i-1}$ if such an $m$ exists and let $g_{i}=0$ otherwise. Also let

$$
M[i, k]=M\left[i-1, k+j(i, k)-q_{i}\right]+1 \text { for } 1 \leqq k \leqq g_{i} .
$$

For those $i$ with $g_{i}>0$, this defines $M[i, k]$ for $1 \leqq k \leqq g_{i}$. If $m=M[i, k]$ for such an $i$ and $k$, let $s_{m}=j(i, k)$; if $m$ is a positive integer not in the image set of $M$, let $s_{m}=\infty$. Also let $s_{0}=\operatorname{deg} \eta+$ $\mu$ wgt $\eta$. Since $M$ is easily shown to be injective, the sequence $\sigma(\pi)=s_{0}, s_{1}, \ldots$ is now well defined. $[\sigma(\rho \eta)$ depends only on $\eta$.]

Let $\sigma(\pi)=s_{0}, s_{1}, \cdots$ and $\sigma\left(\pi^{\prime}\right)=s_{0}^{\prime}, s_{1}^{\prime}, \cdots$. If there is an integer $m$ such that $s_{m}<s_{m}^{\prime}$ and $s_{k}=s_{k}^{\prime}$ for $k<m$, then $\pi$ is said to be stronger than $\pi^{\prime}\left(\pi \gg \pi^{\prime}\right)$ at $m$. The stronger than relation is transitive but is not a complete linear ordering.

3. The set $A$. An $i$-tuple

$$
s_{c n+1}, s_{c n+2}, \cdots, s_{c n+i}
$$

in $\sigma(\pi)$ for which each of these $i$ terms is finite is an i-run for $\pi$ and the sum of the $i$ terms is the weight of the $i$-run. It can be shown that the weight of the $i$-run (2) is a nondecreasing function of $c$ in (2). If the weight of an $n$-run for $\pi$ is in the given set $T$, the associated product

$$
b=y_{1 s_{c n+1}} y_{2 s_{c n+2}} \cdots y_{n s_{c n+n}}
$$

is called a $\beta$-factor of $\pi$. The set $A$ is now defined to consist of all $\pi$ having no $\beta$-factors and the set $C$ to consist of all

$$
\gamma=\alpha \xi, \quad \alpha \in A, \quad \xi=x_{t_{1}} x_{t_{2}} \cdots x_{t_{e}}, \quad e \geqq 1, \quad t_{k} \in T .
$$

In $\S 5, C$ and $L=A \cup C$ will be shown to be space bases for $I$ and $R$, respectively. When $q=0, T=\{0,1, \cdots\}$, and each $v_{t}=0$, $A$ and $C$ can be shown to be the same as the sets of $\alpha$-terms and $\beta$-terms respectively, defined in [5], using the machinery in [7] and induction on $n$.

4. The bijection $\theta$. Next we define a mapping $\theta$ from $P$ to $L$ and, as in Levi's work in [7], show that $\theta$ is a bijection and then show that $L$ and $C$ are space bases for $R$ and $I$, respectively. 
Let $\pi$ have the $b$ of (3) as a $\beta$-factor and let $\sigma(\pi)=s_{0}, s_{1}, \cdots$. It is easily seen that

$$
\sigma(\pi / b)=s_{0}, \cdots, s_{c n}, s_{(c+1) n+1}, \cdots
$$

i.e., that $\sigma(\pi / b)$ is $\sigma(\pi)$ with the $n$-run corresponding to $b$ deleted. This implies that $\pi$ can be written as $\alpha b_{1} b_{2} \cdots b_{r}$, with the $b_{k}$ all the $\beta$-factors of $\pi$ and $\alpha \in A$; then $\theta$ is defined by

$$
\theta(\pi)=\theta\left(\alpha b_{1} b_{2} \cdots b_{r}\right)=\alpha x_{t_{1}} x_{t_{2}} \cdots x_{t_{r}}, \quad \text { where } t_{k}=\text { wgt } b_{k} .
$$

If $\pi$ has no $\beta$-factors, $\pi$ is an $\alpha$ in $A$ and $\theta(\pi)=\theta(\alpha)=\alpha$.

Examination of the sequence $\sigma(\pi)=s_{0}, s_{1}, \cdots$ shows that $\theta$ is injective. Since the terms $\alpha x_{t_{1}} x_{t_{2}} \cdots x_{t_{r}}$ in (5) are easily seen to be in one-to-one correspondence with the $3 n$-section partitions dealt with in [3], Theorem 1 of that paper shows that $\theta$ is a bijection.

\section{The space bases $C$ and $L$.}

LEMMA. If $\pi$ has a $\beta$-factor $b=y_{1 j_{1}} \cdots y_{n j_{n}}$ of weight $t$, then

$$
\pi=f_{0} \pi_{0} x_{t}+f_{1} \pi_{1}+f_{2} \pi_{2}+\cdots+f_{s} \pi_{s}
$$

where $f_{h} \in F, \pi_{h} \gg \pi$, and $\operatorname{deg} \pi_{h}+\operatorname{wgt} \pi_{h} \leqq \operatorname{deg} \pi+\operatorname{wgt} \pi$ for $0 \leqq$ $h \leqq s . \quad A l s o, \operatorname{deg} \pi_{0}=\operatorname{deg} \pi-n$ and $\operatorname{wgt} \pi_{0}=\operatorname{wgt} \pi-t$.

Proof. By definition of $x_{t}$,

$$
x_{t}-v_{t}=e_{0} b+e_{1} b_{1}+\cdots+e_{r} b_{r},
$$

where each $e_{h}$ is a nonzero element of $F$ and for $1 \leqq h \leqq r$,

(7) $b_{h}=y_{1 k_{1}} \cdots y_{n k_{n}}$, with $k_{1}+\cdots+k_{n}=t=j_{1}+\cdots+j_{n}$ and $k_{i} \neq j_{\imath}$ for some $i$.

Solving (6) for $b$ and letting $\pi_{0}=\pi / b$ and $\pi_{h}=\pi_{0} b_{h}$ for $1 \leqq h \leqq r$ yields

$$
\pi=\pi_{0} b=-f_{0} \pi_{0} v_{t}+f_{0} \pi_{0} x_{t}+f_{1} \pi_{1}+\cdots+f_{r} \pi_{r} .
$$

By definition of $v_{t}$, one can write

$$
-f_{0} \pi_{0} x_{t}=f_{r+1} \pi_{r+1}+\cdots+f_{s} \pi_{s}
$$

where, for $r<h \leqq s$, one has $f_{h} \in F$, and $\pi_{h} \gg \pi$ at 0 .

For $1 \leqq h \leqq r, \pi_{h}=\left(\pi b_{h}\right) / b$, with $b_{h}$ as in (7) and so $\operatorname{deg} \pi_{h}=\operatorname{deg} \pi$, wgt $\pi_{h}=$ wgt $\pi$. From (7), it follows that $k_{i}<j_{i}$ for some $i$. Let the $\beta$-factor $b$ of $\pi$ be as in (3). If $k_{1}<j_{i}$, it can be seen that $\pi_{h} \gg \pi$ at some $m$ with $m \leqq c n+1$, and if $k_{i}<j_{i}, i>1$, then 
$\pi_{h} \gg \pi$ at some $m$ with $m \leqq c n$. Since $\pi_{0}=\pi / b$, we have deg $\pi_{0}=$ $\operatorname{deg} \pi-n$, wgt $\pi_{0}=\operatorname{wgt} \pi-t$, and $\pi_{0} \gg \pi$ at 0 .

THEOREM 1. $L$ and $C$ are space bases for $R$ and $I$, respectively.

Proof. Let $B$ be the complement of $A$ in $P$. For every nonnegative integer $s$, let $P(s)$ consist of all $\pi$ in $P$ with $\operatorname{deg} \pi+$ wgt $\pi \leqq s$. Let $R(s)$ be the subspace of $R$ generated by $P(s)$. Let $A(s), B(s), C(s), I(s)$, and $L(s)$ be the intersections with $R(s)$ of $A$, $B, C, I$, and $L$, respectively. Note that $R(s)$ has finite dimension.

The space $R(s)$ is generated by its elements of the form $\pi \xi$, with $\pi$ in $P(s)$ and $\xi$ a $p p$ in the $x_{t}$ with $t$ in $T$, since the elements of this form with $\xi=1$ generate $R(s)$. Since $B(s)$ is finite and the "stronger than" relation is transitive, the lemma implies that $R(s)$ is generated by its elements $\alpha \xi$ with $\alpha$ in $A(s)$ and $\xi$ a $p p$ in the $x_{t}$ with $t$ in $T$, i.e., $L(s)$ generates $R(s)$.

Since $\theta$ with its domain restricted to $B(s)$ is a bijection onto $C(s), L(s)=A(s) \cup C(s)$ has the same finite number of elements as $P(s)=A(s) \cup B(s)$. Since $P(s)$ is a basis for the space $R(s)$, this means that the set $L(s)$ of generators for $R(s)$ is also a basis for $R(s)$. Then it follows that $L$ is a space basis for $R$.

The space $I$ is generated by the $\pi \xi$ with $\pi$ in $P$ and $\xi$ a $p p$ of positive degree in the $x_{t}$ with $t$ in $T$; then $C$ generates $I$ since the $\pi$ in $B$ can be replaced by linear combinations of elements of $L$. Since $C$ is a subset of the basis $L$ for $R$, the elements of $C$ are linearly independent and so $C$ is a basis for $I$.

6. The algorithm $\varphi$. The algorithm $\varphi$ for determining whether a polynomial $r$ of $R$ is in $I$ consists of using the lemma in $\S 5$ to replace in $r$ the $p p$ belonging to $B$ and continuing until $r$ is expressible as

$$
r=f_{1} \alpha_{1} \xi_{1}+\cdots+f_{m} \alpha_{m} \xi_{m}, \quad f_{h} \in F, \quad \alpha_{h} \in A,
$$

with each $\xi_{h}$ a $p p$ in the $x_{t}$ with $t \in T$. Then $r$ is in $I$ if and only if each $\xi_{h}$ has positive degree in the $x_{t}$.

The description of $\varphi$ implies that a nonzero polynomial $r$ of $R$ is not in $I$ if the $p p$ of each term of $r$ is in $A$. This motivates the presentation of some simple sufficient conditions for a $p p \pi$ to be in $A$. First, for a given $Q$, if $\pi=\rho \eta_{1} \eta_{2} \cdots \eta_{n}$, then $\pi$ is in $A$ if $\operatorname{deg} \eta_{i}=0$ for some $i$.

Secondly, let a $p p \pi$ have a function $j(i, k)$ and integers $g_{i}$ as in $\S 2$. The condition 


$$
\begin{gathered}
q_{1}+j(2,1)+\cdots+j(n, 1) \geqq d_{1}+q \\
\left(q=q_{1}+\cdots+q_{n}+q_{n+1}\right)
\end{gathered}
$$

is sufficient for $g_{n}$ to be 0 and hence for $\pi$ to be in $A$. Interchanging 1 and some $h, 1<h \leqq n$, as subscripts $i$ leads to

$$
q_{h}+j(1,1)+\cdots+j(n, 1) \geqq d_{h}+q+j(h, 1) .
$$

If for some fixed $h$ this inequality holds for the $p p$ of all the terms in a nonzero $r$ of $R$, then $r$ is not in $I$.

7. Application to differential ideals. Let $y_{1}, \cdots, y_{n}$ be independent differential indeterminates over a differential field $F$ of characteristic 0 . Let $z=y_{1} y_{2} \cdots y_{n}$, and let $y_{i j}$ and $z_{j}$ be the $j$ th derivatives of $y_{i}$ and $z$, respectively.

For any choice of $q_{1}, q_{2}, \cdots, q_{n}, q_{n+1}$ as nonnegative integers with $q=q_{1}+\cdots+q_{n+1}$ and $T=\{q, q+1, \cdots\}$, the $z_{k}$ meet the conditions required of the $x_{k}$ in $\S 1$ and hence it follows that the set $A$ of $\S 5$ forms an $\alpha$-set for the differential ideal

$$
I=\left[z_{q}\right]=\left(z_{q}, z_{q+1}, \cdots\right)
$$

and hence $\left\{z_{q}\right\}$ is a strong sequence.

8. Combinatorial applications. Let the signature of a $\pi$ in $P$ be the $n$-tuple $E=\left[e_{1}, \cdots, e_{n}\right]$ with $e_{k}$ the degree of $\pi$ in the factors $y_{i j}$ with $i=h$. A polynomial is homogeneous with signature $E$ if it is in the subspace $V[E]$ generated by the $\pi$ of signature $E$. A polynomial is isobaric of weight $w$ if it is in the subspace $V_{w}$ generated by the $\pi$ of weight $w$. Let $V(w, E)=V[E] \cap V_{w}$ and let $p(w, E)$ be the dimension of the subspace $V(w, E)$.

Let $S=\left[s_{1}, s_{2}, \cdots, s_{n}\right]$, with the $s_{i}$ nonnegative integers that are not all zero. A strong sequence $\left\{x_{j}\right\}=x_{q}, x_{q+1}, \ldots$ will be called an S-sequence if:

(i) each $x_{j}$ is homogeneous with signature $S$ and is isobaric with weight $j$, and

(ii) $\left\{x_{j}\right\}$ has an $\alpha$-set consisting of homogeneous and isobaric polynomials.

Let $A$ be such an $\alpha$-set for fixed $S$-sequence $\left\{x_{j}\right\}=x_{q}, x_{q+1}, \cdots$ and let

$$
n_{\alpha}(w, E)=n_{\alpha}\left(w ; e_{1}, \cdots, e_{n}\right)
$$

be the number of elements in $A \cap V(w, E)$. We note here that $n_{\alpha}(w ; 0,0, \cdots, 0)$ equals 1 if $w=0$ and equals 0 if $w>0$.

TheOREm 2. The dimension of the vector space $V(w, E)$ can be expressed as 


$$
p(w, E)=n_{\alpha}(w, E)+\sum n_{\alpha}\left(i ; e_{1}-k s_{1}, \cdots, e_{n}-k s_{n}\right) p(j, k)
$$

where the sum is taken over all nonnegative integers $i, j, k$ such that $i+j+q k=w, k>0$, and the $e_{h}-k s_{h} \geqq 0$ for $1 \leqq h \leqq n$.

Proof. Each side of equation (8) is the dimension of the finite dimensional space $V(w, E)$. The left side is the number of elements in the basis consisting of the $\pi$ in $V(w, E)$ while the right side is the number of $\alpha \xi$, associated with the strong sequence $\left\{x_{j}\right\}$, in $V(w, E)$.

The formula (8) in Theorem 2 enables one to calculate the $n_{\alpha}(w, E)$ recursively, i.e., for a given $E=\left[e_{1}, \cdots, e_{n}\right]$ in terms of values $n_{\alpha}\left(w^{\prime}, E^{\prime}\right)$ with signatures $E^{\prime}=\left[e_{1}^{\prime}, \cdots, e_{n}^{\prime}\right]$ having $e_{h}^{\prime} \leqq e_{h}$ for $1 \leqq h \leqq n$ and $e_{h}^{\prime}<e_{h}$ for some $h$. We next use this to obtain the following:

THEOREM 3. The number $n_{\alpha}(w, E)$ depends only on $w, E, q$, and $S$ and can be written as $n_{\alpha}(w, E, q, S)$.

Proof. For definiteness, let $s_{1}>0$. Then we use induction on $e_{1}$. If $e_{1}=0, n_{\alpha}(w, E)$ clearly depends only on $w, E, q$, and $S$ since $n_{\alpha}(w, E)=p(w, E)$ in this case. By Theorem $2, n_{\alpha}(w, E)=p(w, E)-$ $\sum n_{\alpha}\left(i ; e_{1}-k s_{1}, \cdots, e_{n}-k s_{n}\right) p(j, k)$. Since $k>0$ and $s_{1}>0, e_{1}-$ $k s_{1}<e_{1}$. Now our result follows using the inductive hypothesis on the factors $n_{\alpha}\left(i ; e_{1}-k s_{1}, \cdots, e_{n}-k s_{n}\right)$.

If one has two $S$-sequences (with the same $S$ ) the easiest way to calculate $n_{\alpha}(w, E, q, S)$ for one of the sequences may be to calculate it using the $\alpha$-set for the other sequence (and Theorem 3). Also the identity in (8) can be used to show that a given sequence may not be strong. We next illustrate these two types of applications of Theorems 2 and 3.

First, let $Q=\left\{q_{1}, q_{2}, \cdots, q_{n+1}\right\}$ be a fixed $(n+1)$-tuple of nonnegative integers with $q_{1}+q_{2}+\cdots+q_{n+1}=q=\left(\begin{array}{l}n \\ 2\end{array}\right)$ and let $T=$ $\{q, q+1, \cdots\}$. For $t \in T$, let $x_{t}$ be a linear combination over $F$, of all products $y_{1 j_{1}} \cdots y_{n j_{n}}$ with $j_{1}+\cdots+j_{n}=t$ such that those products with $j_{i} \geqq q_{i}$ for $1 \leqq i \leqq n$ have nonzero coefficients. By the results in $\S 2-5$, the sequence $\left\{x_{j}\right\}$ for the ideal $J=\left(x_{q}, x_{q+1}, \cdots\right)$ is strong, has an $\alpha$-set $A$, and is an $S$-sequence with $S=[1,1, \cdots, 1]$.

Next, let $q=\left(\begin{array}{l}n \\ 2\end{array}\right)$ and $W_{n, q+k}$ be the $k$ th derivative of the Wronskian $W_{n}$ of $n$ independent differential indeterminates $y_{1}, \cdots, y_{n}$. Then $W_{n j}$ is homogeneous with signature $S=[1,1, \cdots, 1]$ and isobaric with weight $j$. Also the work in Mead's paper [9] shows that $\left\{W_{n j}\right\}$ is an $S$-sequence. Hence we have the following: 
THEOREM 4. $J \cap V(w, E)$ and $\left[W_{n}\right] \cap V(w, E)$ have the same dimension given by either side of the equation:

$$
p(w, E)-n_{\alpha}(w, E)=\sum n_{\alpha}\left(i ; e_{1}-k, \cdots, e_{n}-k\right) p(j, k)
$$

where the sum is over all nonnegative integers $i, j, k$ with

$$
i+j+k\left(\begin{array}{l}
n \\
2
\end{array}\right)=w
$$

and

$$
1 \leqq k \leqq m=\min \left\{e_{1}, \cdots, e_{n}\right\}
$$

In [3], the author stated that Theorem 4 could be proved and used this result to obtain many identities on combinatorial generating functions.

Secondly, Theorem 2 can be used to show that a given sequence $\left\{x_{j}\right\}$ is not strong, and hence to indicate the existence of syzygies. For example, consider the differential ideal $[x]=\left(x_{2}, x_{3}, \cdots\right)$ where $x_{2+j}$ is the $j$ th derivative of $x=y_{0}^{2} y_{2}+7 y_{0} y_{1}^{2}$. The recursive calculation, using (8), of $n_{\alpha}(w, E)$ based on the assumption that $\left\{x_{j}\right\}$ is strong leads to the contradiction that the cardinality of $A \cap V(w, E)$ is negative for some $w$ and $E$. The following is a partial printout from a computer program designed to compute $n_{\alpha}(w, E, q, S)$ and $p(w, E)-n_{\alpha}(w, E, q, S)$ [denoted by $n_{\alpha}$ and $n_{\beta}$ resp. in the table] for this example where we have

$$
n=1, \quad q=2, \quad S=\left[s_{1}\right]=[3] .
$$

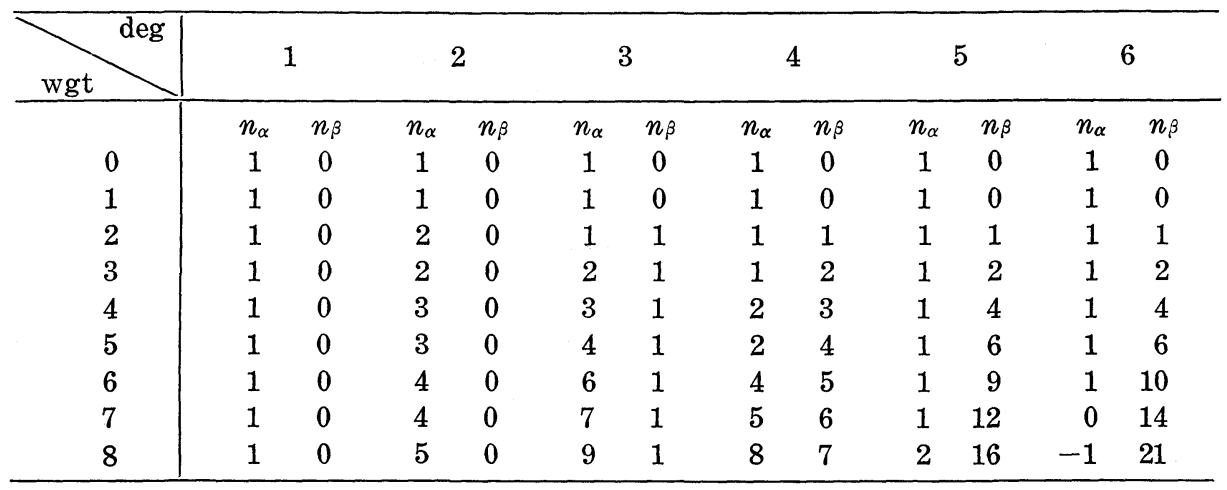

The negative entry -1 for $n_{\alpha}$ in the table for weight 8 and degree 6 shows that no sequence $\left\{x_{j}\right\}$ satisfying properties (9) is strong, and also indicates that any such sequence has some syzygies involving only polynomials with the weight bounded by 8 and the degree bounded by 6 . 
9. Bibliography, The many fields of mathematics in which tableaux and skew-tableau play an important role are described in the papers of the report [2]. The set of all linear combinations of partitions is shown to be isomorphic to the differential polynomial ring in one indeterminate in [4]. The ordered pairs of generalized tableaux used by Mead in [9] appear in a more general setting in [1]. The different proof of Mead's Theorem 2 in that paper could be eliminated by a reference to D. Knuth's generalization of the Robinson-Schensted insertion into tableau algorithm in [6]. The ordering of power products described in $\$ 2$ above made possible the generalization, given here, of the results of [5] and [7].

\section{REFERENCES}

1. P. Doubilet, G. C. Rota and J. Stein, On the foundations of combinatorial theory: IX combinatorial methods in invariant theory, Studies in Applied Mathematics, Vol. LIII, No. 3, September 1974, 185-216.

2. D. Foata, ed., Combinatoire et Représentation du Groupe Symétrique (Actes de la Table Ronde du C. N. R. S. tenue a l'Université Louis-Pasteur de Strasbourg, du 26 au 30 avril 1976).

3. R. M. Grassl, Multisectioned partitions of integers, Pacific J. Math., 69 (1977), 415-424.

4. A. P. Hillman and R. M. Grassl, Polynomial ring generated by tableau shapes, European Journal of Combinatorics, to appear.

5. A. P. Hillman, D. G. Mead, K. B. O'Keefe and E. S. O'Keefe, Ideals generated by products, Proc. Amer. Math. Soc., 17 (1966), 717-719.

6. D. E. Knuth, Permutations, matrices and generalized Young tableaux, Pacific J. Math., 34 (1970), 709-727.

7. H. Levi, On the structure of differential polynomials and on their theory of ideals, Trans. Amer. Math Soc., 51 (1942), 532-568.

8. D. G. Mead, Differential ideals, Proc. Amer. Math. Soc., 6 (1955), 420-432.

9. - Determinantal ideals, identities and the Wronskian, Pacific J. Math., 42 (1972), 165-175.

10. D. G. Mead and B. D. McLemore, Ritt's question on the Wronskian, Pacific J. Math., 35 (1970), 467-472.

Received August 9, 1977 and in revised form January 27, 1981.

The University of New Mexico

Albuquerque, NM 87131 



\section{PACIFIC JOURNAL OF MATHEMATICS}

EDITORS

DONALD BABBITT (Managing Editor)

University of California

Los Angeles, CA 90024

Hugo RossI

University of Utah

Salt Lake City, UT 84112

C. C. MOORE and ANDREW OGG

University of California

Berkeley, CA 94720

\section{J. DugundjI}

Department of Mathematics

University of Southern California

Los Angeles, CA 90007

R. FINN and J. MILGRAM

Stanford University

Stanford, CA 94305

\section{ASSOCIATE EDITORS}
R. ARENS
E. F. BECKENBACH
B. H. NEUManN
F. WOLF
K. YoSHIDA

\section{SUPPORTING INSTITUTIONS}

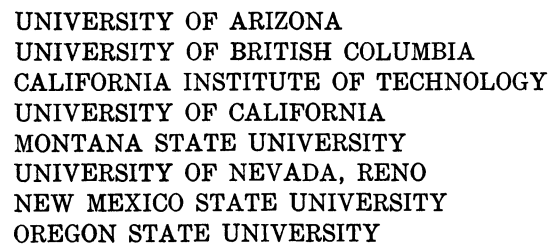

UNIVERSITY OF ARIZONA

UNIVERSITY OF BRITISH COLUMBIA

CALIFORNIA INSTITUTE OF TECHNOLOGY

UNIVERSITY OF CALIFORNIA

MONTANA STATE UNIVERSITY

UNIVERSITY OF NEVADA, RENO

NEW MEXICO STATE UNIVERSITY

OREGON STATE UNIVERSITY

\author{
UNIVERSITY OF OREGON \\ UNIVERSITY OF SOUTHERN CALIFORNIA \\ STANFORD UNIVERSITY \\ UNIVERSITY OF HAWAII \\ UNIVERSITY OF TOKYO \\ UNIVERSITY OF UTAH \\ WASHINGTON STATE UNIVERSITY \\ UNIVERSITY OF WASHINGTON
}

The Supporting Institutions listed above contribute to the cost of publication of this Journal, but they are not owners or publishers and have no responsibility for its content or policies.

Mathematical papers intended for publication in the Pacific Journal of Mathematics should be in typed form or offset-reproduced, (not dittoed), double spaced with large margins. Please do not use built up fractions in the text of the manuscript. However, you may use them in the displayed equations. Underline Greek letters in red, German in green, and script in blue. The first paragraph or two must be capable of being used separately as a synopsis of the entire paper. Please propose a heading for the odd numbered pages of less than 35 characters. Manuscripts, in triplicate, may be sent to any one of the editors. Please classify according to the scheme of Math. Reviews, Index to Vol. 39. Supply name and address of author to whom proofs should be sent. All other communications should be addressed to the managing editor, or Elaine Barth, University of California, Los Angeles, California, 90024.

50 reprints to each author are provided free for each article, only if page charges have been substantially paid. Additional copies may be obtained at cost in multiples of 50 .

The Pacific Journal of Mathematics is issued monthly as of January 1966. Regular subscription rate: $\$ 102.00$ a year (6 Vols., 12 issues). Special rate: $\$ 51.00$ a year to individual members of supporting institutions.

Subscriptions, orders for numbers issued in the last three calendar years, and changes of address shoud be sent to Pacific Journal of Mathematics, P.O. Box 969, Carmel Valley, CA 93924, U.S.A. Old back numbers obtainable from Kraus Periodicals Co., Route 100, Millwood, NY 10546.

\section{PUBLISHED BY PACIFIC JOURNAL OF MATHEMATICS, A NON-PROFIT CORPORATION}

Printed at Kokusai Bunken Insatsusha (International Academic Printing Co., Ltd.). 8-8, 3-chome, Takadanobaba, Shinjuku-ku, Tokyo 160, Japan. 


\section{Pacific Journal of Mathematics}

\section{Vol. 97, No. $2 \quad$ February, 1981}

Patrick Robert Ahern and N. V. Rao, A note on real orthogonal measures . . . . . 249

Kouhei Asano and Katsuyuki Yoshikawa, On polynomial invariants of fibered

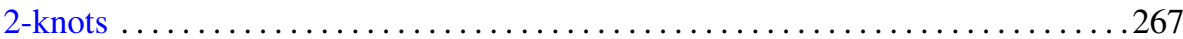

Charles A. Asmuth and Joe Repka, Tensor products for $S L_{2}(\mathscr{K})$. I.

Complementary series and the special representation

Gary Francis Birkenmeier, Baer rings and quasicontinuous rings have a

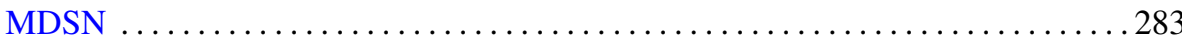

Hans-Heinrich Brungs and Günter Törner, Right chain rings and the generalized

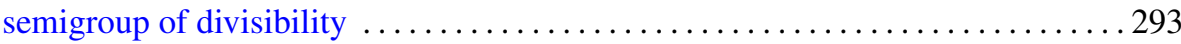

Jia-Arng Chao and Svante Janson, A note on $H^{1} q$-martingales . . . . . . . . . 307

Joseph Eugene Collison, An analogue of Kolmogorov's inequality for a class of

additive arithmetic functions

Frank Rimi DeMeyer, An action of the automorphism group of a commutative ring on its Brauer group

H. P. Dikshit and Anil Kumar, Determination of bounds similar to the Lebesgue

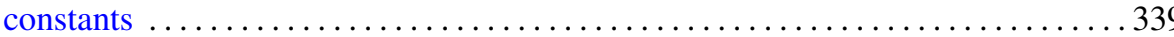

Eric Karel van Douwen, The number of subcontinua of the remainder of the plane

D. W. Dubois, Second note on Artin's solution of Hilbert's 17th problem. Order spaces

Daniel Evans Flath, A comparison of the automorphic representations of GL(3) and its twisted forms

Frederick Michael Goodman, Translation invariant closed $*$ derivations

Richard Grassl, Polynomials in denumerable indeterminates

K. F. Lai, Orders of finite algebraic groups

George Kempf, Torsion divisors on algebraic curves

Arun Kumar and D. P. Sahu, Absolute convergence fields of some triangular matrix methods

Elias Saab, On measurable projections in Banach spaces

Chao-Liang Shen, Automorphisms of dimension groups and the construction of AF algebras

Barry Simon, Pointwise domination of matrices and comparison of $\Phi_{p}$ norms

Chi-Lin Yen, A minimax inequality and its applications to variational inequalities

Stephen D. Cohen, Corrections to: "The Galois group of a polynomial with two indeterminate coefficients"

Phillip Schultz, Correction to: "The typeset and cotypeset of a rank 2 abelian group"

Pavel G. Todorov, Correction to: "New explicit formulas for the $n$th derivative of composite functions"

Douglas S. Bridges, Correction to: "On the isolation of zeroes of an analytic function" 